\title{
ATAC-Seq Reveals the Landscape of Open Chromatin and cis-Regulatory Elements in the Phytophthora sojae Genome
}

\author{
Zhichao Zhang, ${ }^{1,2,3}$ Long Lin,, ${ }^{1,2,3}$ Han Chen, ${ }^{1,2,3}$ Wenwu Ye, ${ }^{1,2,3, \dagger}$ Suomeng Dong, ${ }^{1,2,3}$ Xiaobo Zheng, ${ }^{1,2,3}$ \\ and Yuanchao Wang ${ }^{1,2,3}$ \\ ${ }^{1}$ Department of Plant Pathology, Nanjing Agricultural University, Nanjing, Jiangsu 210095, China \\ ${ }^{2}$ Key Laboratory of Plant Immunity, Nanjing Agricultural University, Nanjing, Jiangsu 210095, China \\ ${ }^{3}$ Key Laboratory of Integrated Management of Crop Diseases and Pests (Ministry of Education), Nanjing, Jiangsu 210095, China \\ Accepted 12 January 2022.
}

\begin{abstract}
Nucleosome-free open chromatin often harbors transcription factor (TF)-binding sites that are associated with active cisregulatory elements. However, analysis of open chromatin regions has rarely been applied to oomycete or fungal plant pathogens. In this study, we performed the assay for transposaseaccessible chromatin with high-throughput sequencing (ATACseq) to identify open chromatin and cis-regulatory elements in Phytophthora sojae at the mycelial stage. We identified 10,389 peaks representing nucleosome-free regions (NFRs). The peaks were enriched in gene-promoter regions and associated with $40 \%$ of $P$. sojae genes; transcription levels were higher for genes with multiple peaks than genes with a single peak and were higher for genes with a single peak than genes without peak. Chromatin accessibility was positively correlated with gene transcription level. Through motif discovery based on NFR peaks in core promoter regions, 25 candidate cis-regulatory motifs with evidence of TF-binding footprints were identified. These motifs exhibited various preferences for location in the promoter region and associations with the transcription level of their target genes, which included some putative pathogenicityrelated genes. As the first study revealing the landscape of open chromatin and the correlation between chromatin accessibility and gene transcription level in oomycetes, the results provide a technical reference and data resources for future studies on the regulatory mechanisms of gene transcription.
\end{abstract}

Keywords: ATAC-seq, cis-regulatory element, oomycete, open chromatin, promoter, transcription factor

Chromatin structure is an important factor for transcriptional regulation in eukaryotic organisms. Open chromatin in the

${ }^{\dagger}$ Corresponding author: W. Ye; yeww@njau.edu.cn

Funding: This work was supported by grants from the National Natural Science Foundation of China (31772140 and 31721004).

*The $\boldsymbol{e}$-Xtra logo stands for "electronic extra" and indicates there are supplementary figures and tables published online.

The author(s) declare no conflict of interest.

(c) (1) ()$\Theta$ Copyright $(\odot) 2022$ The Author(s). This is an open access article distributed under the CC BY-NC-ND 4.0 International license. promoter, enhancer, and other gene regulatory regions allows for binding of transcription factors (TFs), which regulate gene transcription (Thurman et al. 2012). With recent advancements in high-throughput sequencing, a series of omics technologies have been developed for identifying open chromatin, e.g., formaldehyde-assisted isolation of regulatory elements (Giresi et al. 2007), micrococcal nuclease digestion with deep sequencing (Kent et al. 2011), methylated DNA immunoprecipitation sequencing (Kelly et al. 2012), DNase I-hypersensitive site sequencing (DNase-seq) (Thurman et al. 2012), and assay for transposase-accessible chromatin with high-throughput sequencing (ATAC-seq) (Buenrostro et al. 2013). Compared with other open chromatin sequencing technologies, ATAC-seq is faster and more efficient. It can be used to identify regions of open and nucleosome-bound chromatin and to infer the binding positions of DNA/TF-binding proteins through footprinting analysis. For these reasons, ATAC-seq has been widely used in recent years for large-scale identification of open chromatin in mammals and plants (Bao et al. 2015; Lu et al. 2017; Murano et al. 2018; Pálfy et al. 2020; Starks et al. 2019); however, this method has only been applied to a few fungi, including Ustilaginoidea virens, Sprassis latifolia, and Aspergillus spp. (Chen et al. 2021; Huang et al. 2021; Yang et al. 2019), and there was no report in oomycetes.

Phytophthora sojae is a filamentous oomycete plant pathogen that causes soybean stem and root rot, causing serious yield reductions in soybean production worldwide (Kamoun et al. 2015; Tyler et al. 2006). Precise gene transcriptional regulation is essential to the development and infection processes of $P$. sojae. For example, transcription of genes encoding RxLR effectors, which target various functional processes of plant defense responses, is programmed globally (Wang et al. 2011). In the field population of $P$. sojae, differences in transcription of the avirulence genes PsAvrla and PsAvr $3 a$ result in gain or loss of virulence (Qutob et al. 2009). PsAvr3b was engineered using mutated promoters, which led to differences in transcription levels and plant disease compatibility in $P$. sojae (Ochola et al. 2020). Silencing of PSGPAl, which encodes a G-protein alpha subunit in the cellular signaling pathway, does not impair hyphal growth or sporulation but severely affects zoospore behavior (Hua et al. 2008). Transcription of PsSAKl, a gene encoding a stress-activated mitogen-activated protein kinase, is upregulated in zoospores, cysts, and during early infection; silencing of PSSAK1 severely hinders zoospore development and pathogenicity (Li et al. 2010). Several genes of the Myb TF family are 
differentially transcribed in response to the silencing of PSSAK1, while silencing of PSMYB1 affects zoospore development (Zhang et al. 2012).

TFs play important roles in oomycete biological processes through regulation of gene transcription at various stages. In $P$. infestans, some Myb genes are upregulated at the sporangium and zoospore stages and, thus, may be related to spore development (Xiang and Judelson 2010); PiMADS, a MADSbox TF-encoding gene, is necessary for asexual sporulation (Leesutthiphonchai and Judelson 2018); overexpression of the CBF/NFY gene PITG_10861 results in increased synthesis of $\alpha$ hormone (Wang et al. 2020); and the bZIP TF PiBZP1 is a key regulator in zoospore-mediated infection (Blanco and Judelson 2005). In both $P$. sojae and $P$. litchii, the bZIP TF BZP32 is involved in cyst germination, the oxidative stress response, and pathogenicity (Kong et al. 2020; Sheng et al. 2021). In P. sojae, the heat-shock TF PsHSF1 helps pathogens manage oxidative stress by reducing the accumulation of plant-produced reactive oxygen species (Sheng et al. 2015), while the MADS-box TF PsMAD1 is involved in zoosporogenesis and pathogenesis (Lin et al. 2018b). Recently, the "plant-specific" RWP-RK TF family was found in oomycetes, and some genes showed specific conserved transcription patterns during development and pathogenesis (Yin et al. 2020).

Given the major roles of TFs in transcriptional regulation, identification of TF-binding sites (TFBS) is crucial to elucidating their functional mechanisms. The first systematic TFBS discovery analysis in oomycetes utilized the genome sequences of $P$. infestans and two related Phytophthora species ( $P$. ramorum and $P$. sojae) along with in planta gene transcription data and predicted 19 conserved DNA motifs (Seidl et al. 2012). Then, genome-wide prediction of promoter motifs in $P$. infestans revealed 103 motifs, and stage-specific transcription patterns were validated for some motifs, using the $G U S$ reporter gene (Roy et al. 2013a). The absence of a TATA box and presence of novel core promoter elements in P. infestans and other oomycetes were also reported (Roy et al. 2013b). However, all of these candidate TFBS were initially identified based on bioinformatics-based prediction using artificially defined regions as putative promoter sequences. Because the promoter region is variable in length and some gene models may be incorrect, the accuracy of the promoter sequences used for motif discovery was not perfect.

In this study, we confirmed that ATAC-seq was feasible for identification of open chromatin and TFBS in the $P$. sojae genome. We identified 10,389 peaks representing nucleosomefree open chromatin regions. These peaks were enriched in gene promoter regions, and the chromatin accessibility of these peaks showed a positive correlation with the transcription level of their target genes. Motif discovery analysis revealed 25 TF-binding footprint-supported cis-regulatory elements in core promoter regions. Location preference and association with gene transcription level were also investigated for these motifs.

\section{RESULTS}

\section{Identification of the nucleosome-free open chromatin regions.}

To identify open chromatin and cis-regulatory elements in the $P$. sojae genome (Fig. 1A), we generated approximately 20 million sequence reads through ATAC-seq, using DNA from mycelia for library construction. After read processing, read mapping, and alignment processing, 4,728,346 highquality reads (17.0\% of clean reads) were obtained for subsequent analysis (Fig. 1B). The distribution of sequence fragment size showed obvious periodicity, with three major regions indicating a nucleosome-free region (NFR) $(<100 \mathrm{bp})$, a mononucleosome protected region (MNR) (150 to $250 \mathrm{bp}$ ), and a di-nucleosome protected region ( $>250$ bp) (Fig. 2A). The distributions of the NFR and MNR fragments were enriched in the region upstream of the start codon (ATG), whereas the signal for MNR fragments showed two peaks surrounding the single peak of NFR fragments (Fig. 2B). Using the NFR fragments, we identified 10,389 NFR peaks (Fig. 1B; Supplementary Table S1). Almost $70 \%$ and over $60 \%$ of the peaks, respectively, were enriched within 1 and $0.5 \mathrm{~kb}$ upstream of the ATG (Fig. $2 \mathrm{C}$ ); thus, we defined $0.5 \mathrm{~kb}$ upstream of the ATG as the core promoter region for further analysis. Promoter-flanking genomic regions contained fewer chromatin accessible peaks, suggesting that such regions might be bound and protected by nucleosomes (Fig. 2B and C). These results indicate that the ATAC-seq assay in $P$. sojae was successful.

\section{Gene transcription level is correlated} with chromatin accessibility.

Among 19,230 annotated $P$. sojae genes, 7,652 (40\%) genes were associated with NFR peaks, including 5,724 genes associated with one peak, and 1,406 and 522 genes associated with two and more than two peaks, respectively (Fig. 2D). Through analysis of the relationship between the transcription level of target genes and the number of peaks, we found that genes associated with one or more peaks exhibited significantly higher transcription levels than genes without a peak (Fig. 3A), indicating that the chromatin-accessible regions were involved in transcriptional activation of their target genes. In addition, transcription levels were significantly higher for genes associated with two or more peaks than genes associated with a single peak (Fig. 3A).

According to the number of $\operatorname{Tn} 5$ transposase cut sites in peaks, chromatin accessibility could be evaluated. Among the genes associated with one or more peaks, the proportion of those with detectable transcription increased gradually as chromatin accessibility increased, with Pearson's correlation coefficient $R=0.86(P<0.05)$ (Supplementary Fig. S1). Through analysis of the relationship between chromatin accessibility of peaks and transcription level of target genes of peaks, we found a positive correlation, with $R=0.33(P<0.01)$ (Fig. 3B). These results suggest that chromatin accessibility in the promoter region is positively correlated with the transcription level of target genes in $P$. sojae.

Gene transcription is controlled by the interplay of regulatory events at promoters and distal regulatory elements called enhancers. We analyzed the genes that contain one peak inside (I-peak) and one peak outside (O-peak) the core promoter regions and found that the transcription levels of these genes were significantly higher than the genes that appear to contain a single I-peak or a single O-peak (Fig. 3C). The single I-peaks and single O-peaks both showed lower chromatin accessibility than the I-peaks that have an additional O-peak (Fig. 3C). These results imply that the O-peaks might contain enhancers, to increase chromatin accessibility of the corresponding I-peaks, as well as transcription level of the target genes.

\section{Identification of the footprint-supported cis-regulatory elements.}

To assess potential TFBSs or cis-regulatory elements, we performed motif discovery analysis using 6,248 NFR peaks identified in the core promoter region (Fig. 1B). Using the DREME tool in MEME Suite (Bailey et al. 2015), we identified 56 candidate motifs of 5 to $8 \mathrm{nt}$ in length. Through the FIMO search program, we then reproduced the distributions of 48 motifs in the NFR regions and filtered out eight redundant motifs. The 48 motifs 
were designated ATAC motif 1 to 48 (AM-1 to AM-48); 65\% of these motifs $(n=31)$ were similar to motifs previously predicted in $P$. infestans, based on genome-wide promoter sequence analysis (Roy et al. 2013a; Seidl et al. 2012) (Table 1).

ATAC-seq can be used for analysis of TF-binding footprints, as TFs bind to DNA to protect it from $\mathrm{Tn} 5$ transposase cleavage (Fig. 1A). Among the 48 motifs analyzed, 25 motifs exhibited strong footprint characteristics (Fig. 4A and B; Supplementary
Fig. S2), which were considered as candidate cis-regulatory elements. Most of these motifs (22 motifs, 88\%) were similar to motifs previously reported in $P$. infestans (Table 1).

\section{Motif location preference and association} with gene transcription.

Most of the 25 footprint-supported motifs in the core promoter were located within $150 \mathrm{bp}$ upstream of the start codon, with a

\section{A}

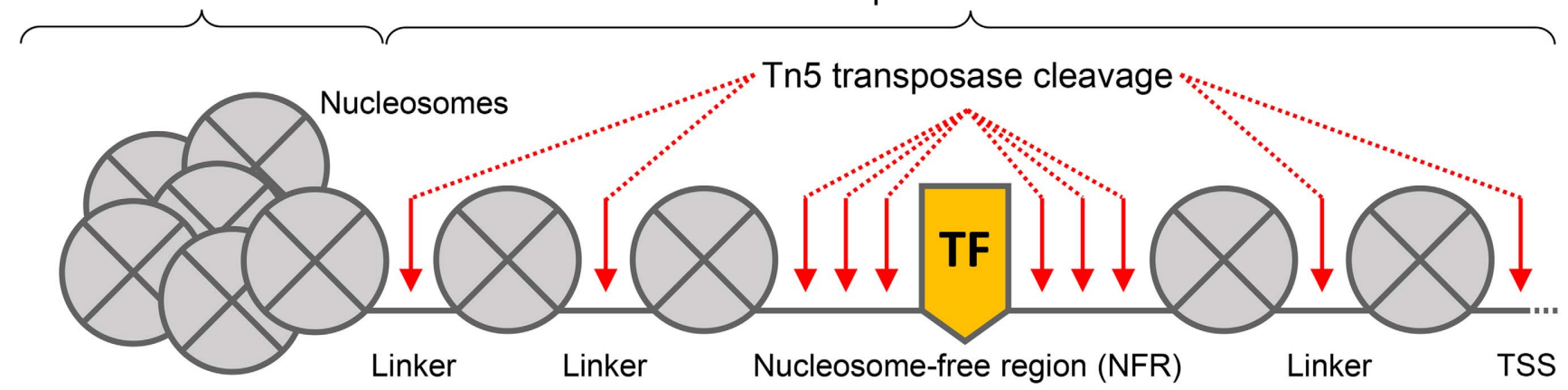

ATAC fragments (red: nucleosome-free fragments)
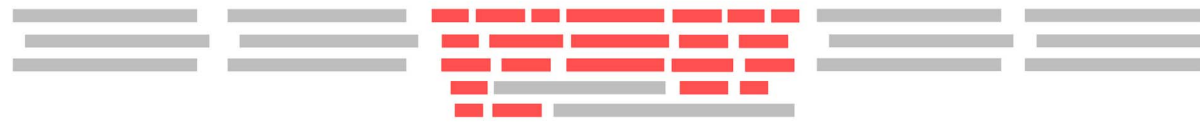

Fragment density

Nucleosome-free peak

Footprint

B

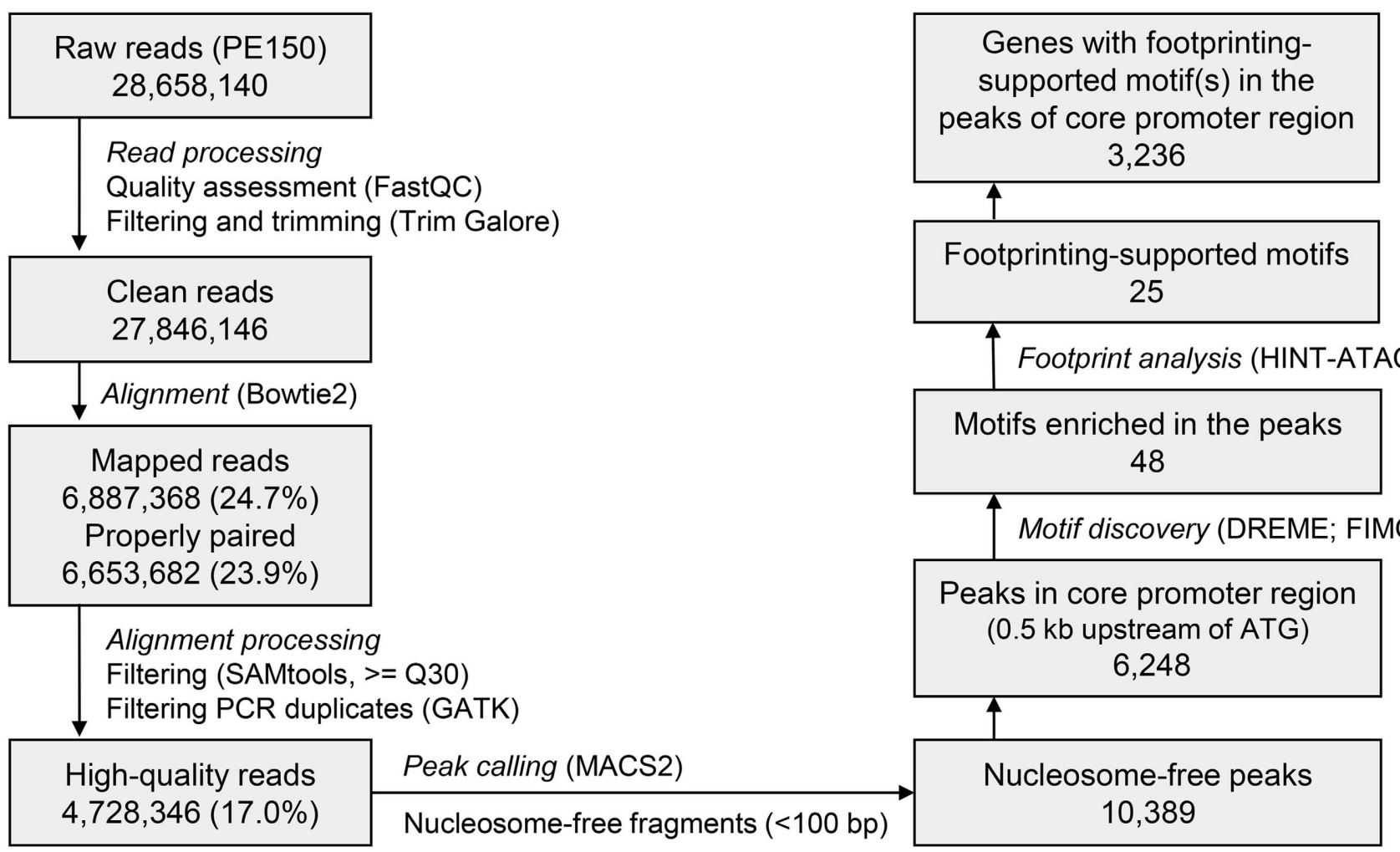

Fig. 1. Biological principle and data analysis flow chart of ATAC-seq in this study. A, Cleavage sites of Tn5 transposase in open chromatin regions, the sequence reads obtained in this study, and a nucleosome-free peak with a transcription factor (TF)-binding footprint. B, Data analysis flow chart and corresponding results are shown at each step. 
frequency of $58 \%$. Different motifs showed differing location preferences, with four, 11, and 10 motifs concentrated in the ranges of -150 to $-100 \mathrm{bp},-100$ to $-50 \mathrm{bp}$, and -50 to $-1 \mathrm{bp}$, respectively (Fig. 5A). The NFR peaks containing different motifs exhibited differences in chromatin accessibility, and genes associated with different motifs had differing transcription levels. A strong positive correlation relationship was observed between the chromatin accessibility of NFR peaks located in motifs and transcription levels of motif-associated genes $(R=0.76, P<0.01)$ (Fig. 5B and
C). We also observed that the NFR peaks and genes containing motifs near the start codon had higher chromatin accessibility and transcription levels, respectively (Fig. 5A, B, and C).

A total of $3,236 P$. sojae genes contained one or more footprint-supported motifs in the core promoter region, and 711 genes were predicted to function as pathogenicity-related genes, including genes encoding secreted proteins (SPs), effectors (EFs), carbohydrate-active enzymes, oxidoreductases, transporters, protein kinases (PKs), and TFs (Supplementary Table S2). Probably
A
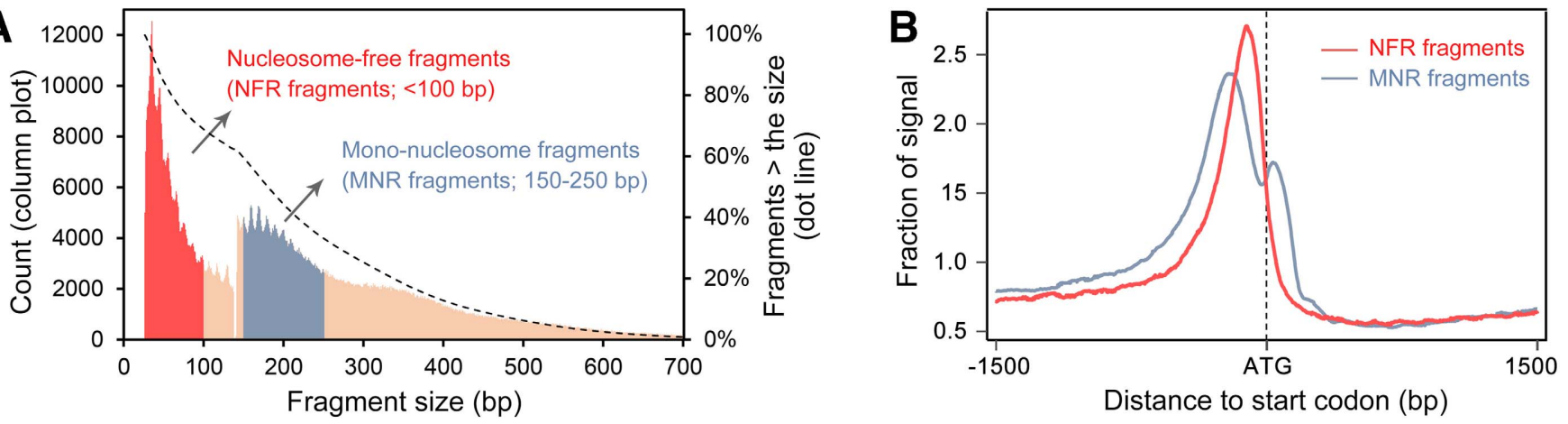

C

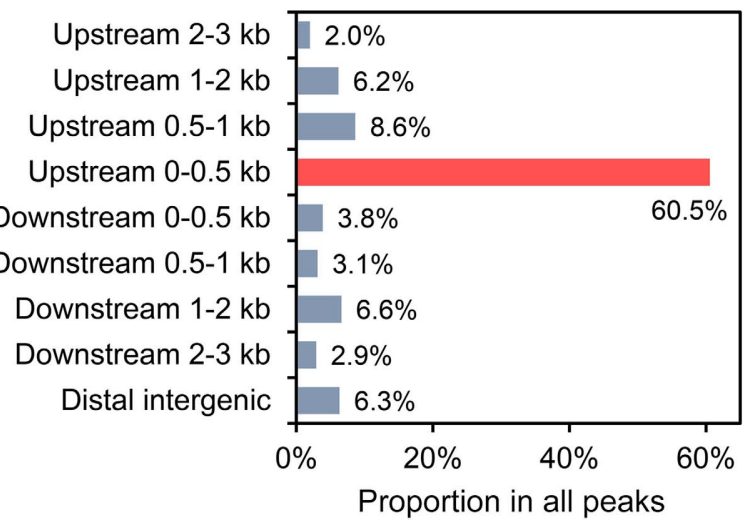

D

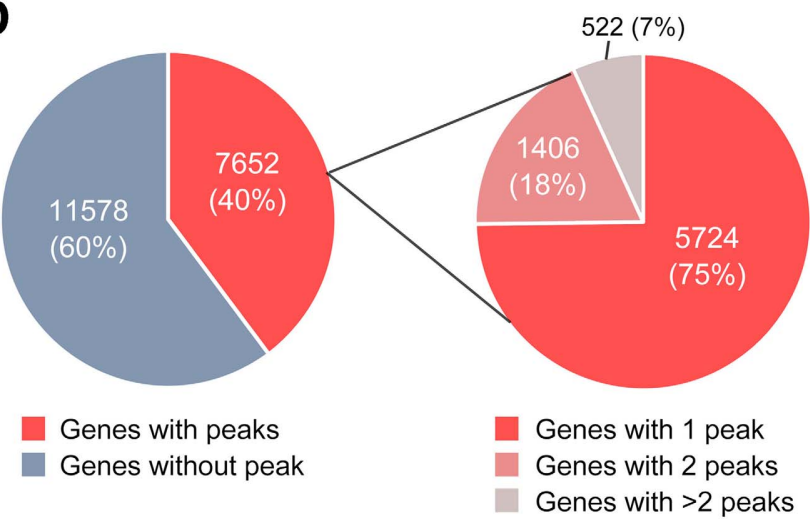

Fig. 2. Characteristics of sequence fragments and peaks obtained from ATAC-seq analysis. A, Distribution of ATAC-seq fragment sizes. NFR = nucleosome-free region, $\mathrm{MNR}=$ mononucleosome protected region. B, Distribution of nucleosome-free fragments and mononucleosome fragments surrounding the gene start codon. $\mathbf{C}$, Distribution of nucleosome-free peaks surrounding the gene start codon. D, Statistics of genes associated with different numbers of peaks.
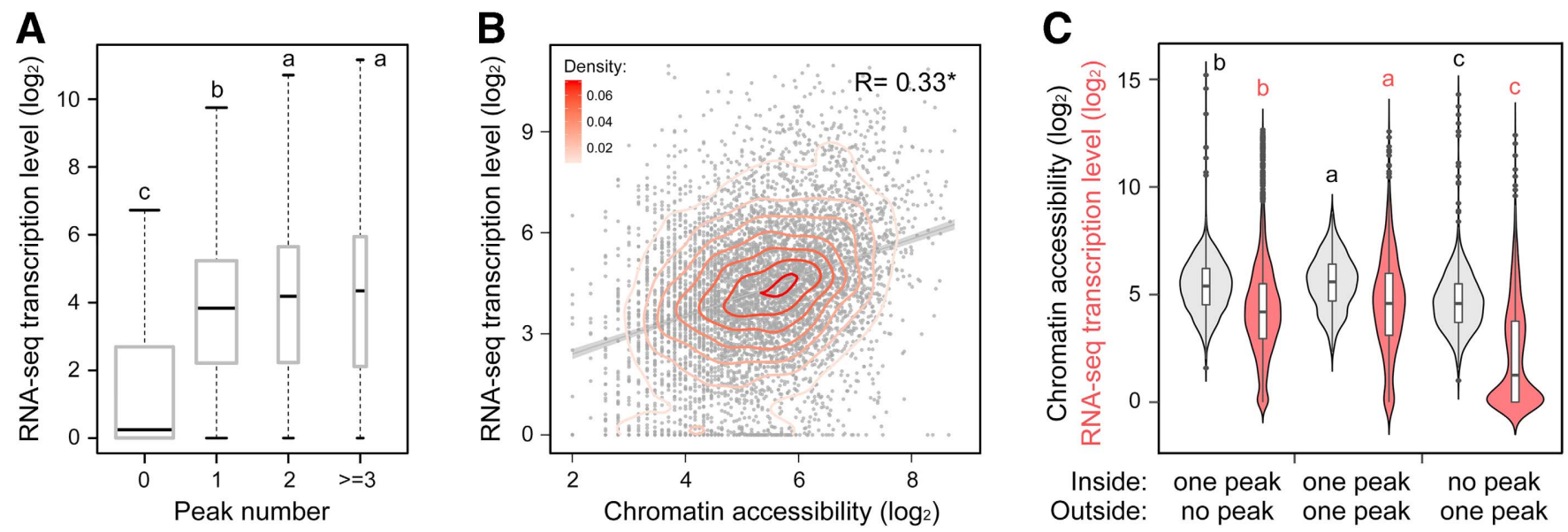

Fig. 3. Correlations of peak number and chromatin accessibility with gene transcription level. A, Comparison of gene transcription levels according to peak number. B, Correlation analysis between gene transcription level and chromatin accessibility. The Pearson correlation coefficient $(R)$ is shown, with the asterisk indicating $P<0.01$. RNA-seq data from Phytophthora sojae at the mycelial stage were used for analysis. C, Comparison of gene transcription levels and chromatin accessibility among three categories of genes: $1+0,1+1$, and $0+1$, based on the number of peaks inside the core promoter (first digit) and outside the core promoter (final digit). 
because ATAC-seq was performed at the $P$. sojae mycelial stage, motif-associated genes showed relatively high average transcription levels at this stage (Fig. 5C). Comparing the proportion of genes containing one or more motifs among pathogenicityrelated gene families, we found that the $\mathrm{PK}$ gene family accounted for the largest proportion, while the SP and EF families made up smaller proportions (Fig. 5D); it is well-known that many genes in the SP and EF families are specifically induced during infection.

\section{DISCUSSION}

ATAC-seq has been widely used to identify open chromatin in animals and plants (Davie et al. 2015; Lu et al. 2017; Maher et al. 2018; Starks et al. 2019), but probably due to the difficulty of application in microorganisms, few studies have used this method in filamentous plant pathogens. Contamination of DNA from bacteria and organelles (e.g., mitochondria) is a common problem, which can result in a low mapping rate of reads. To identify the source of contaminants for our data, we performed k-mer analysis using Kraken2 (Wood and Salzberg 2014) and found that $16 \%$ of the clean reads were mapped to bacteria and $83 \%$ of the clean reads had no hits. We actually performed two ATAC-seq assays with and without adding antibiotics for culturing $P$. sojae mycelia, respectively, and found that adding antibiotics is useful to improve the read mapping rate. Further efforts are still needed to identify the causes of contamination and reduce the contamination.

Table 1. Motifs predicted from the nucleosome-free region peaks at core promoter region

\begin{tabular}{|c|c|c|c|}
\hline Motif ID ${ }^{a}$ & Sequence pattern & Motifs in publication $1^{b}$ & Motifs in publication $2^{c}$ \\
\hline AM-1 (FP) & [GA]CCAATC[GA] & M40: CAATCAG & M4: CCAAT \\
\hline AM-2 (FP) & [GA]CTTCAA[CG] & $\begin{array}{l}\text { M57: CTTC[AG]AC; } \\
\text { M58: CTTCAAC }\end{array}$ & $\begin{array}{l}\text { M2: ACTTCAAC; } \\
\text { M15: [ATG][CA]TTCAACCA }\end{array}$ \\
\hline AM-3 (FP) & ATGG[CAG]TAC & M36: ATGGCTAC & - \\
\hline AM-4 & ATACAAA & - & M3: ATACAAANNCAAGTC \\
\hline AM-5 (FP) & TAC[TC]G[TA]A & $\begin{array}{l}\text { M4: [ACG]TAC[ACT]GTA; } \\
\text { M87: T[AG]C[AT]GTAC }\end{array}$ & - \\
\hline AM-6 (FP) & GTG[GCT]ACCA & M83: GTGC[AT]GCA & - \\
\hline AM-7 (FP) & CG[TC]ACTTA & M53: CTCACTTC & - \\
\hline AM-8 (FP) & T>GTGT[GA] & - & M18: T[ACG]GTGTGG[TC] \\
\hline AM-9 (FP) & GCTGCTG[CG] & M75: GCTGC[AT]G & - \\
\hline AM-10 (FP) & [GA]CCAATG[GA] & M40: CAATCAG & - \\
\hline AM-11 & CGCCGC[CG] & M47: CGCCACC & - \\
\hline AM-12 (FP) & A[ATG]AGTGA & - & - \\
\hline AM-13 (FP) & C[TC][TC]CCTC & $\begin{array}{l}\text { M54: CTCCTTC; } \\
\text { M60: CTTCT[TC]C }\end{array}$ & - \\
\hline AM-14 & CCAATC $[\mathrm{CA}]$ & M40: CAATCAG & - \\
\hline AM-15 & ACTTG[GA][TC] & M25: ACT[CT]G[AGC]AG & - \\
\hline AM-16 (FP) & [CG]AAG[TA]GA & M3: [ACG]AAGT[ACG]G & - \\
\hline AM-17 & CCTA[CG]CCC & - & - \\
\hline AM-18 & GCCAG[TC]C & - & - \\
\hline AM-19 (FP) & TACCGGT[TA] & $\begin{array}{l}\text { M88: T[AG]CCGG[TC]A; } \\
\text { M94: TACCGGTA }\end{array}$ & M9: GTACCGGTA \\
\hline AM-20 (FP) & TACATGTA & M93: TACATGTA & M1: TACATGTA[CT] \\
\hline AM-21 (FP) & CGCCAT[CG]G & - & - \\
\hline AM-22 & GACG[ACT]CGA & M24: ACGCCGG & - \\
\hline AM-23 & CTTCC[ACT]GC & - & - \\
\hline AM-24 (FP) & C[ATG]CCACCA & M5: [AT]CCACCA & - \\
\hline AM-25 & GAGACCG[TA] & - & - \\
\hline AM-26 & AAAAAG[TA] & M14: AAAAGAAG & - \\
\hline AM-27 & AAAACCT & - & - \\
\hline AM-28 (FP) & CGCCAC[GA][TC] & M47: CGCCACC & - \\
\hline AM-29 & ATCTTG[GA] & - & - \\
\hline AM-30 & CCATCAC[TC] & M41: CATC[ACG]A[CT]G & - \\
\hline AM-31 (FP) & GAGCGAG[CG] & - & - \\
\hline AM-32 & TACTACTA & M80: GTACTAC & - \\
\hline AM-33 & ATAGAG[GA]G & M30: AGAGAGAG & - \\
\hline AM-34 (FP) & CCAAGCAG & M19: AAGCA[AG]A & - \\
\hline AM-35 & GACG[ATG]GGC & M18: AACGGGGT & - \\
\hline AM-36 (FP) & CCGAG[TC]C & - & - \\
\hline AM-37 & CC[ATG]TTTC & - & - \\
\hline AM-38 & CGTC[CA]GTC & - & - \\
\hline AM-39 & ACCGACC[CAG] & - & - \\
\hline AM-40 (FP) & GCAGCG[CA]C & M70: GCA[AG]TGC & - \\
\hline AM-41 & TCTGAT[TA] & - & - \\
\hline AM-42 (FP) & TGGCT[ATG]CA & M98: TG[CG]CTG[CG]C & - \\
\hline AM-43 & AAGATCGC & - & - \\
\hline AM-44 (FP) & GC[TC]GGCC & - & - \\
\hline AM-45 (FP) & CG[GCT]CGCAG & M48: CGCCGAAG & - \\
\hline AM-46 & TATT[TA]ATA & M89: T[AT]TTAATA & M7: TA[CT]TATTAATA \\
\hline AM-47 (FP) & TGGAC[CAG]AA & - & - \\
\hline AM-48 & ATATTT[GA]G & M37: ATT[CT]TTA & - \\
\hline
\end{tabular}

\footnotetext{
${ }^{\mathrm{a}} \mathrm{FP}$ indicates the footprint-supported motifs.

${ }^{\mathrm{b}}$ Roy et al. (2013a) has been designated publication 1, 103 predicted motifs.

${ }^{\mathrm{c}}$ Seidl et al. (2012) has been designated publication 2, 22 predicted motifs.
} 
According to the periodic distribution of fragment sizes, the major parts of sequence fragments obtained from ATAC-seq could be readily divided into NFR and MNR fragments. MNR is a mononucleosome-binding region and less accessible to TF binding, while NFR is a nucleosome-free region and a TF would probably bind; thus we focused on NFR for TF-binding motif discovery. We found that the NFRs were most enriched near the transcription initiation site, which is similar to the previous reports (Buenrostro et al. 2013; Lu et al. 2017). Although the RNA-seq data were not generated alongside the ATAC data for $P$. sojae at the mycelial stage, there was a positive correlation between chromatin accessibility and the transcription level of target genes of peaks, consistent with those reported in mammals (Ampuja et al. 2017; Pastor et al. 2014; Starks et al. 2019). The above evidence suggested that ATAC-seq can be applied to $P$. sojae and may also be applicable to more oomycete and fungal pathogens.

The main benefit of ATAC-seq is the ability to identify TF footprints similar to DNase-seq, as the cleavage preference of $\mathrm{Tn} 5$ transposase is low ( $\mathrm{Li}$ et al. 2019; Yan et al. 2020). In this study, we used HINT-ATAC software to correct for Tn5 cleavage preference ( $\mathrm{Li}$ et al. 2019). Through footprinting analysis of 48 motifs enriched by DREME, we observed that 25 motifs exhibited apparent footprint characteristics. The 25 footprintsupported motifs identified here may be helpful for accurately identifying regulatory regions and factors in $P$. sojae (Neph et al. 2012; Sung et al. 2016). Low sequencing depth or low native chromatin accessibility affect the detection of footprints, which may be one reason for the footprints of other motifs not being observed (Quach and Furey 2017). In addition, because TF binding is an instantaneous and transient status, some motif sites are difficult to be captured in Tn5 cleavage experiments (Gusmao et al. 2016; Sung et al. 2014). We also found that the distal peaks additional to the core promoter peaks may contain enhancers. Additional evidence of enhancers may be obtained through combined analysis with other methods, such as epigenetic assays for $\mathrm{H} 3 \mathrm{~K} 4 \mathrm{me} 1$ and H3K27ac and highest-throughput chromosome conformation capture (Hi-C) assay (Lin et al. 2018a; RadaIglesias et al. 2011), and further experimental verification.

Using the Tomtom tool in MEME Suite (Bailey et al. 2015), we found that several motifs had matches in the database of known motifs (Supplementary Table S3). For example, AM-1 had a match to nuclear transcription factor $\mathrm{Y}$ subunit alpha (NFYA). NFYA is a key subunit of the nuclear TF trimer complex, which specifically binds to DNA sequences and functions as a regulatory subunit. NFY forms a highly conserved TF that binds to CCAAT motifs in the promoter regions of many genes. In Fusarium graminearum, CCAAT-binding TFs play important roles in development, DNA damage response, virulence, and toxin production (Kim et al. 2020). Recent research has
A
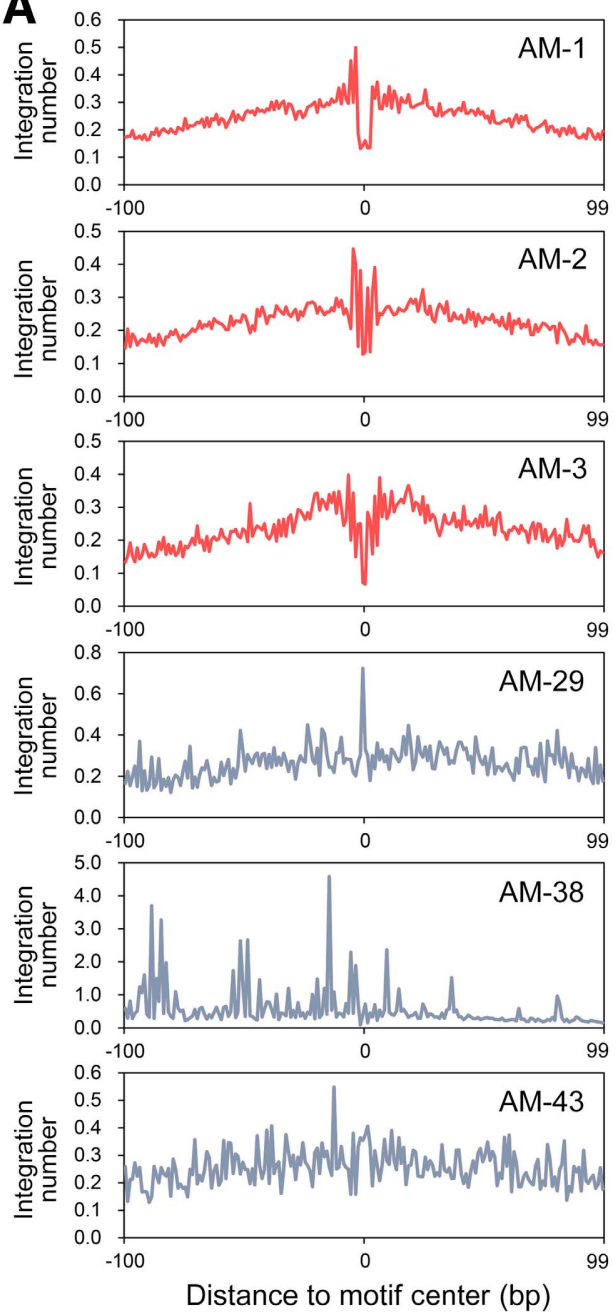

B

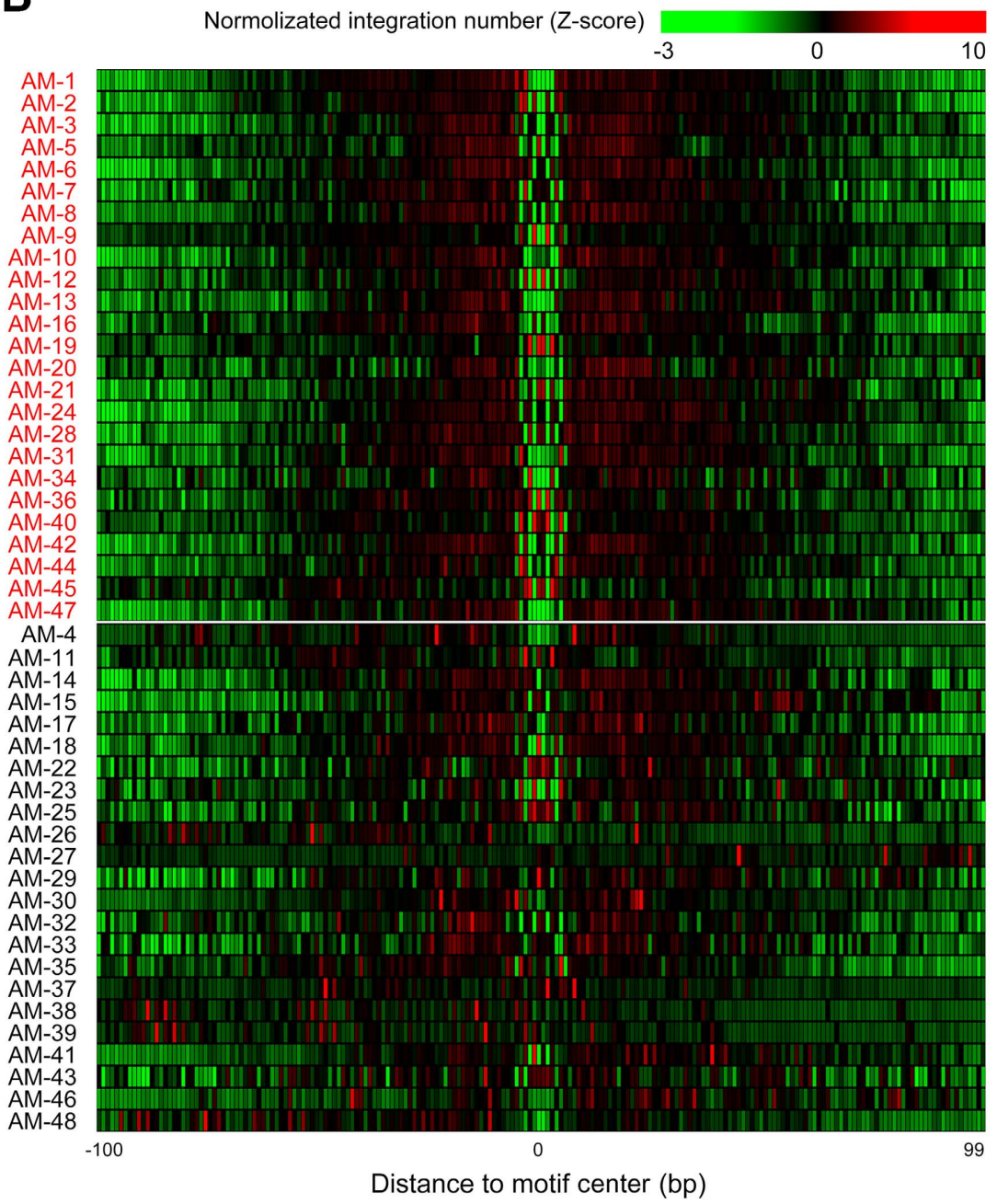

Fig. 4. Transcription factor (TF)-binding footprint analysis. A, Examples of motifs with (red) and without (grayd) apparent footprint characteristics based on Tn5 integration sites. Typical TF-binding footprint profile exhibits specific signal fluctuations (usually weakening) at the motif center. B, Distribution of Tn5 transposase integration number surrounding the centers of 48 motifs. Motifs with apparent footprint profiles, designated highly reliable motifs, are highlighted with red labels. 
demonstrated that overexpression of a TF related to $\mathrm{CBF} / \mathrm{NFY}$ in $P$. infestans increases the synthesis of $\alpha$ hormone (Wang et al. 2020); thus, NFY may also play an important regulatory role in $P$. sojae. AM-2 has an apparent footprint but unknown annotation in databases. However, AM-2 was previously predicted in $P$. infestans (Table 1) and revealed as footprint-supported motifs in $P$. sojae in this study, suggesting that this motif may be conserved to Phytophthora species.

We have observed TF-binding footprints at the promoter region of some pathogenicity-related genes. Further ATAC-seq analysis would be useful to reveal the dynamics of open chromatin regions and chromatin accessibility of these genes and to identify the cisregulatory elements associated with their specific transcription at the infection stage. We actually also conducted ATAC-seq assays for $P$. sojae at its infection stage; however, likely due to contamination of DNA from plant tissues and environmental microorganisms such as bacteria, the read-mapping rate was low and the background noise was intense. Therefore, the ATAC-seq data from this stage was not used for further analysis.

In conclusion, our results show that ATAC-seq can be effectively applied to $P$. sojae at the mycelial stage and can be used to identify cis-regulatory elements that are footprint-supported, providing candidates for experimental validation of proteinDNA interactions.

\section{MATERIALS AND METHODS}

Sample preparation, library construction, and sequencing.

To avoid bacterial contamination, ampicillin $(50 \mu \mathrm{g} / \mathrm{ml})$ and rifampicin $(50 \mu \mathrm{g} / \mathrm{ml})$ were added to $P$. sojae (strain P6497) culture medium. After culturing $P$. sojae on a V8 plate at $25^{\circ} \mathrm{C}$ in the dark for 3 days, five $3 \times 3 \mathrm{~mm}$ pieces of mycelial block were incubated, in a culture dish containing V8 liquid medium, in the dark for 3 days, and then, the mycelia were collected. ATAC-seq was performed as previously described (Wu et al. 2016). Mycelia (0.1 g) was ground with liquid nitrogen, was transferred to $1.5-\mathrm{ml}$ precooled NPB (20 mM HEPES, $40 \mathrm{mM}$ $\mathrm{NaCl}, 90 \mathrm{mM} \mathrm{KCl}, 2 \mathrm{mM}$ EDTA, $0.5 \mathrm{mM}$ EGTA, $0.5 \mathrm{mM}$ spermidine, $0.2 \mathrm{mM}$ spermine, and $1 \times$ Roche complete protease inhibitors), and was lysised for $15 \mathrm{~min}$, and then, filtering the mixture through a double-layer of Miracloth, collecting the liquid in a new centrifuge tube, and centrifuging at $6,000 \times g$ for $10 \mathrm{~min}$ at $4^{\circ} \mathrm{C}$. The supernatant was removed, the pellet was resuspended with $1 \mathrm{ml}$ of precooled NEB2 $(0.25 \mathrm{M}$ sucrose, $10 \mathrm{mM}$ Tris- $\mathrm{HCl}, \mathrm{pH} 8.0,10 \mathrm{mM} \mathrm{MgCl}_{2}, 1 \%$ Triton X-100, and $1 \times$ Roche complete protease inhibitors), and the supernatant was transferred to a $1.5-\mathrm{ml}$ centrifuge tube and was centrifuged at $12,000 \times g$ for $10 \mathrm{~min}$ at $4^{\circ} \mathrm{C}$. The supernatant was removed and the pellet was resuspended with $300 \mu \mathrm{l}$ of NEB3 $(95 \%$ (vol/vol) percoll, $0.25 \mathrm{M}$ sucrose, $10 \mathrm{mM}$ Tris- $\mathrm{HCl}, \mathrm{pH} 8.0$, $10 \mathrm{mM} \mathrm{MgCl} 2,5 \mathrm{mM} \beta$-ME, and $1 \times$ Roche complete protease inhibitors). Precooled NEB3 (300 $\mu$ l) was added to a new 1.5-ml centrifuge tube, the supernatant from the previous step was then carefully added to the centrifuge tube, centrifuging at $2,200 \times g, 4^{\circ} \mathrm{C}$ for $10 \mathrm{~min}$. The supernatant was remove and the pellet was resuspended with $1 \mathrm{ml}$ of precooled NPB. Isolated nuclei were stained with $0.04 \%$ trypan blue and were counted using a microscope. Supernatant with 50,000 nuclei from the previous step was added to a new $1.5-\mathrm{ml}$ centrifuge tube, was centrifuged at $500 \times \mathrm{g}, 4^{\circ} \mathrm{C}$ for $10 \mathrm{~min}$, and the

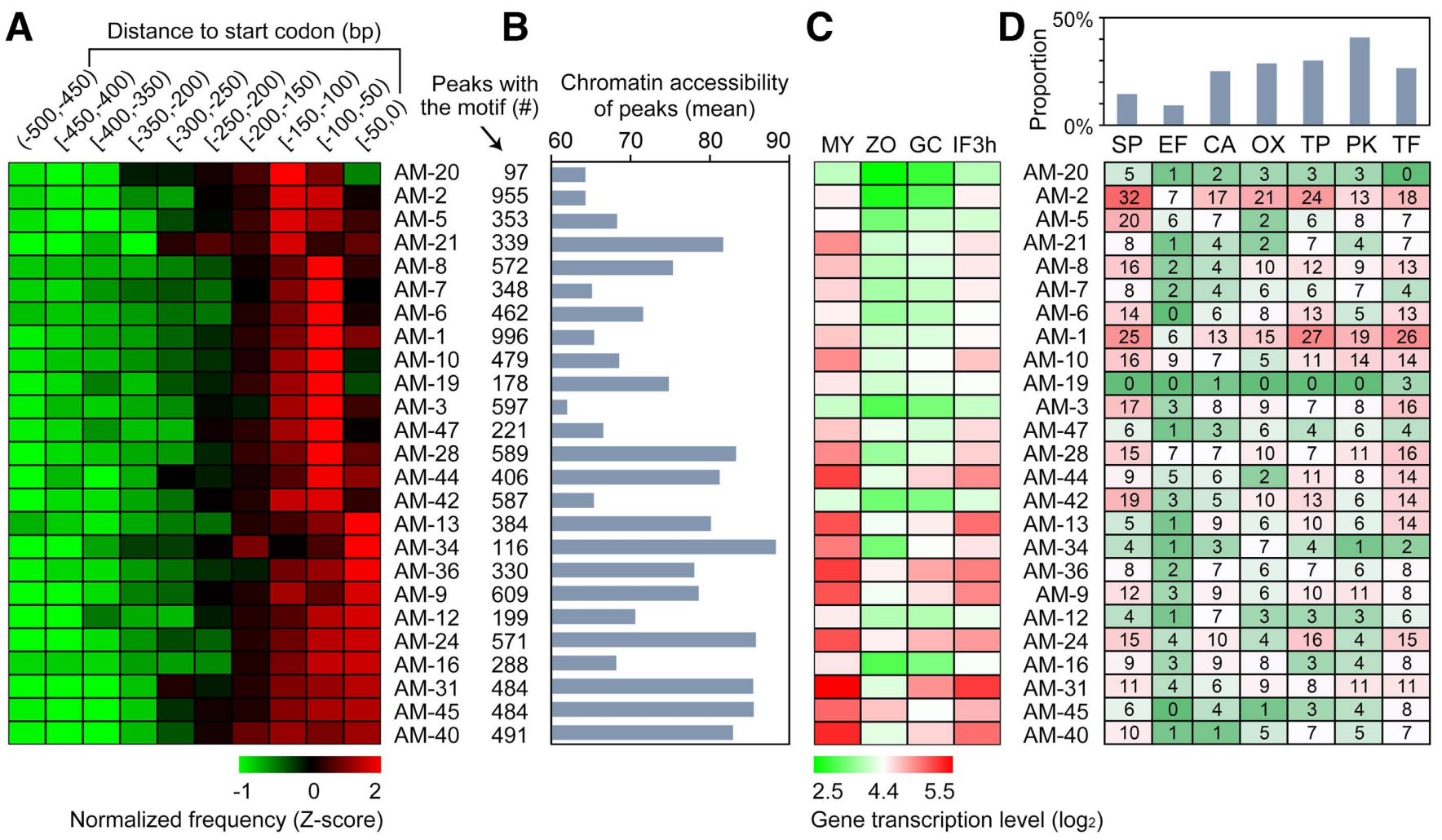

Fig. 5. Characteristics of the 25 footprint-supported motifs. A, Location distribution of motifs in the region $0.5 \mathrm{~kb}$ upstream of the gene start codon. B, The number of nucleosome-free region (NFR) peaks containing the indicated motifs is shown to the left of column plots showing the average chromatin accessibility of the NFR peaks. C, Average transcription levels of genes associated with the indicated motifs. RNA-seq data from Phytophthora sojae at four stages, including mycelia (MY), zoospores (ZO), germinated cysts (GC), and 3 h postinoculation on susceptible soybean leaves (IF3h) are shown. D, Heat map with labels indicating the numbers of motif-associated genes in the indicated gene families $(\mathrm{SP}=\mathrm{secreted}$ protein, $\mathrm{EF}=\mathrm{effector}$, $\mathrm{CA}=$ carbohydrate-active enzyme, $\mathrm{OX}=$ oxidoreductase-related protein, $\mathrm{TP}=$ transporter, $\mathrm{PK}=$ protein kinase, $\mathrm{TF}=$ transcription factor). Upper column plots summarize the proportions of members associated with highly reliable motifs in each gene family. 
supernatant was removed. The pellet was mixed with transposition reaction mixture and was incubated for $30 \mathrm{~min}$ at $37^{\circ} \mathrm{C}$. The transposition reaction mixture contained $25 \mu \mathrm{l}$ of target DNA ( $2 \times$ reaction buffer), $2.5 \mu$ of TDE1 (Nextera Tn 5 transposase), and $22.5 \mu \mathrm{l}$ of nuclease-free $\mathrm{H}_{2} \mathrm{O}$. After the transposition reaction, the Qiagen MinElute PCR purification kit was used immediately to purify DNA. Sequencing library construction was conducted by Igenebook Biotechnology Co., Ltd. (Wuhan, China) using the HiSeq X Ten platform and 150-bp paired-end sequencing.

\section{Data processing.}

Raw reads were filtered with Trim Galore software (version 0.6.6) to remove low-quality reads and adaptor sequences. Clean reads were mapped to the reference genome of $P$. sojae (v1.1) using Bowtie2 software (version 2.2.9) (Langmead and Salzberg 2012). Contaminants was evaluated by Kraken2 (version 2.1.2) (Wood and Salzberg 2014). PCR duplicated reads were removed using GATK software (version 4.1) (McKenna et al. 2010). Using SAMtools software (version 1.3, -f 2 -q 30), only properly paired alignments with alignment quality $\geq 30$ were retained. Then, the reads were shifted $+4 \mathrm{bp}$ and $-5 \mathrm{bp}$ for the positive and negative strand, respectively, using deepTools software (version 3.3, alignment -ATAC shift) (Ramírez et al. 2014). Reads were separated into NFR ( $<100 \mathrm{bp}$ ) and MNR (150 to $250 \mathrm{bp}$ ), based on fragment size, using a custom shell script. The NFR fragments were used to identify open peaks using MACS2 software (version 2.1.4, -f BAMPE -keep-dup all -cutoff-analysis) (Zhang et al. 2008). A fragment size distribution plot was generated with the GATK software. The NFR and MNR fragment distribution around the start codon ATG was plotted with the R package soGGi (version 3.13). ChIPseeker software (version 3.11) was used to assign the center of each peak to the nearest or overlapping gene (Yu et al. 2015).

\section{Correlation between ATAC-seq and RNA-seq results.}

The correlation between chromatin accessibility and the transcription level of target genes was visualized as a density contour plot using the $\mathrm{R}$ package ggplot2 and the function stat_density $2 \mathrm{~d}()$. Because Tn5 transposase cuts two DNA strands with 9-bp overhangs and the adjusted position represents the center point between these cuts, this position is recorded as the cut site representing the chromatin accessibility event. Chromatin accessibility was evaluated based on the number of Tn5 transposase cut sites in peaks, which were calculated by pyDNase software (version 0.2.4, dnase_cut_counter.py -A) and logarithm (base 2) normalized (Piper et al. 2015). RNA-seq data for $P$. sojae at four stages, including mycelia, zoospores, germinated cysts, and $3 \mathrm{~h}$ postinoculation on susceptible soybean leaves, were reported previously (BioProject accession number in GenBank: PRJNA426510) (Wang et al. 2018). RNA-seq results were calculated as fragments per kilobase per million mapped fragments. The Pearson correlation coefficient was calculated using $\mathrm{R}$ packages with the cor.test() function.

\section{Motif discovery and footprint analysis.}

Motif enrichment analysis was performed using the MEME Suite software (version 5.3; including DREME, FIMO, and Tomtom) (Bailey et al. 2015). Peaks in the core promoter region (500 to $1 \mathrm{bp}$ upstream of ATG) were used as the input file of DREME for motif discovery with minimum and maximum widths of 5 and 8, respectively. FIMO was used to determine the positions of motifs and matches were filtered using default parameters $(P$ value $<1 \mathrm{E}-4)$. FIMO automatically filtered several low-credibility motifs and 48 motifs were retained. Tomtom was used to compare the motifs against a database of known motifs with default parameters.
HINT-ATAC corrected for strand-specific Tn5 cleavage bias and was used to predict all footprint sites for NFR peaks according to a typical footprint feature (peak-dip-peak). The footprint profiles were generated for each of the 48 motifs using HINTATAC, with the configuration file customized by modifying the RGT data folder.

\section{Data and code availability.}

The ATAC-seq data have been deposited in GenBank (BioProject accession number: PRJNA761250). ATAC-seq workflow and custom scripts are available in the GitHub repository.

\section{AUTHOR-RECOMMENDED INTERNET RESOURCE}

GitHub repository: https://github.com/biogeeker/atac_analysis

\section{LITERATURE CITED}

Ampuja, M., Rantapero, T., Rodriguez-Martinez, A., Palmroth, M., Alarmo, E. L., Nykter, M., and Kallioniemi, A. 2017. Integrated RNAseq and DNase-seq analyses identify phenotype-specific BMP4 signaling in breast cancer. BMC Genomics 18:68.

Bailey, T. L., Johnson, J., Grant, C. E., and Noble, W. S. 2015. The MEME suite. Nucleic Acids Res. 43 (W1):W39-W49.

Bao, X., Rubin, A. J., Qu, K., Zhang, J., Giresi, P. G., Chang, H. Y., and Khavari, P. A. 2015. A novel ATAC-seq approach reveals lineagespecific reinforcement of the open chromatin landscape via cooperation between BAF and p63. Genome Biol. 16:284.

Blanco, F. A., and Judelson, H. S. 2005. A bZIP transcription factor from Phytophthora interacts with a protein kinase and is required for zoospore motility and plant infection. Mol. Microbiol. 56:638-648.

Buenrostro, J. D., Giresi, P. G., Zaba, L. C., Chang, H. Y., and Greenleaf, W. J. 2013. Transposition of native chromatin for fast and sensitive epigenomic profiling of open chromatin, DNA-binding proteins and nucleosome position. Nat. Methods 10:1213-1218.

Chen, X., Liu, H., Chen, X., Huang, J., Hsiang, T., and Zheng, L. 2021 ATAC-seq data for genome-wide profiling of transcription factor binding sites in the rice false smut fungus Ustilaginoidea virens. Mol. Plant-Microbe Interact 34:830-834.

Davie, K., Jacobs, J., Atkins, M., Potier, D., Christiaens, V., Halder, G., and Aerts, S. 2015. Discovery of transcription factors and regulatory regions driving in vivo tumor development by ATAC-seq and FAIREseq open chromatin profiling. PLoS Genet. 11:e1004994.

Giresi, P. G., Kim, J., McDaniell, R. M., Iyer, V. R., and Lieb, J. D. 2007. FAIRE (formaldehyde-assisted isolation of regulatory elements) isolates active regulatory elements from human chromatin. Genome Res. 17:877-885.

Gusmao, E. G., Allhoff, M., Zenke, M., and Costa, I. G. 2016. Analysis of computational footprinting methods for DNase sequencing experiments. Nat. Methods 13:303-309.

Hua, C., Wang, Y., Zheng, X., Dou, D., Zhang, Z., Govers, F., and Wang, Y. 2008. A Phytophthora sojae G-protein alpha subunit is involved in chemotaxis to soybean isoflavones. Eukaryot. Cell 7:2133-2140.

Huang, L., Li, X., Dong, L., Wang, B., and Pan, L. 2021. Profiling of chromatin accessibility identifies transcription factor binding sites across the genome of Aspergillus species. BMC Biol. 19:189.

Kamoun, S., Furzer, O., Jones, J. D. G., Judelson, H. S., Ali, G. S., Dalio, R. J. D., Roy, S. G., Schena, L., Zambounis, A., Panabières, F., Cahill, D., Ruocco, M., Figueiredo, A., Chen, X. R., Hulvey, J., Stam, R., Lamour, K., Gijzen, M., Tyler, B. M., Grünwald, N. J., Mukhtar, M. S., Tomé, D. F. A., Tör, M., Van Den Ackerveken, G., McDowell, J., Daayf, F., Fry, W. E., Lindqvist-Kreuze, H., Meijer, H. J. G., Petre, B., Ristaino, J., Yoshida, K., Birch, P. R. J., and Govers, F. 2015. The top 10 oomycete pathogens in molecular plant pathology. Mol. Plant Pathol. 16:413-434.

Kelly, T. K., Liu, Y., Lay, F. D., Liang, G., Berman, B. P., and Jones, P. A. 2012. Genome-wide mapping of nucleosome positioning and DNA methylation within individual DNA molecules. Genome Res. 22: 2497-2506.

Kent, N. A., Adams, S., Moorhouse, A., and Paszkiewicz, K. 2011. Chromatin particle spectrum analysis: A method for comparative chromatin structure analysis using paired-end mode next-generation DNA sequencing. Nucleic Acids Res. 39:e26.

Kim, J. E., Nam, H., Park, J., Choi, G. J., Lee, Y. W., and Son, H. 2020 Characterization of the CCAAT-binding transcription factor complex in the plant pathogenic fungus Fusarium graminearum. Sci. Rep. 10:4898. 
Kong, G. H., Chen, Y. B., Deng, Y. Z., Feng, D. N., Jiang, L. Q., Wan, L., Li, M. H., Jiang, Z. D., and Xi, P. G. 2020. The basic leucine zipper transcription factor PIBZP32 associated with the oxidative stress response is critical for pathogenicity of the lychee downy blight oomycete Peronophythora litchii. mSphere 5:e00261-20.

Langmead, B., and Salzberg, S. L. 2012. Fast gapped-read alignment with Bowtie 2. Nat. Methods 9:357-359.

Leesutthiphonchai, W., and Judelson, H. S. 2018. A MADS-box transcription factor regulates a central step in sporulation of the oomycete Phytophthora infestans. Mol. Microbiol. 110:562-575.

Li, A., Wang, Y., Tao, K., Dong, S., Huang, Q., Dai, T., Zheng, X., and Wang, Y. 2010. PsSAK1, a stress-activated MAP kinase of Phytophthora sojae, is required for zoospore viability and infection of soybean. Mol. Plant-Microbe Interact 23:1022-1031.

Li, Z., Schulz, M. H., Look, T., Begemann, M., Zenke, M., and Costa, I. G. 2019. Identification of transcription factor binding sites using ATAC-seq. Genome Biol. 20:45

Lin, D., Hong, P., Zhang, S., Xu, W., Jamal, M., Yan, K., Lei, Y., Li, L., Ruan, Y., Fu, Z. F., Li, G., and Cao, G. 2018a. Digestion-ligation-only $\mathrm{Hi}-\mathrm{C}$ is an efficient and cost-effective method for chromosome conformation capture. Nat. Genet. 50:754-763.

Lin, L., Ye, W., Wu, J., Xuan, M., Li, Y., Gao, J., Wang, Y., Wang, Y., Dong, S., and Wang, Y. 2018b. The MADS-box transcription factor PsMAD1 is involved in zoosporogenesis and pathogenesis of Phytophthora sojae. Front. Microbiol. 9:2259.

Lu, Z., Hofmeister, B. T., Vollmers, C., DuBois, R. M., and Schmitz, R. J. 2017. Combining ATAC-seq with nuclei sorting for discovery of cis-regulatory regions in plant genomes. Nucleic Acids Res. 45:e41.

Maher, K. A., Bajic, M., Kajala, K., Reynoso, M., Pauluzzi, G., West, D. A., Zumstein, K., Woodhouse, M., Bubb, K., Dorrity, M. W. Queitsch, C., Bailey-Serres, J., Sinha, N., Brady, S. M., and Deal, R. B. 2018. Profiling of accessible chromatin regions across multiple plant species and cell types reveals common gene regulatory principles and new control modules. Plant Cell 30:15-36.

McKenna, A., Hanna, M., Banks, E., Sivachenko, A., Cibulskis, K., Kernytsky, A., Garimella, K., Altshuler, D., Gabriel, S., Daly, M., and DePristo, M. A. 2010. The genome analysis toolkit: A MapReduce framework for analyzing next-generation DNA sequencing data. Genome Res. 20:1297-1303.

Murano, K., Iwasaki, Y. W., and Siomi, H. 2018. Profiling open chromatin structure in the ovarian somatic cells using ATAC-seq. Methods Mol. Biol. 1680:165-177.

Neph, S., Vierstra, J., Stergachis, A. B., Reynolds, A. P., Haugen, E., Vernot, B., Thurman, R. E., John, S., Sandstrom, R., Johnson, A. K., Maurano, M. T., Humbert, R., Rynes, E., Wang, H., Vong, S., Lee, K., Bates, D., Diegel, M., Roach, V., Dunn, D., Neri, J., Schafer, A., Hansen, R. S., Kutyavin, T., Giste, E., Weaver, M., Canfield, T., Sabo, P., Zhang, M., Balasundaram, G., Byron, R., MacCoss, M. J., Akey, J. M., Bender, M. A., Groudine, M., Kaul, R., and Stamatoyannopoulos, J. A. 2012. An expansive human regulatory lexicon encoded in transcription factor footprints. Nature 489:83-90.

Ochola, S., Huang, J., Ali, H., Shu, H., Shen, D., Qiu, M., Wang, L., Li, X., Chen, H., Kange, A., Qutob, D., and Dong, S. 2020. Editing of an effector gene promoter sequence impacts plant-Phytophthora interaction. J. Integr. Plant Biol. 62:378-392.

Pálfy, M., Schulze, G., Valen, E., and Vastenhouw, N. L. 2020. Chromatin accessibility established by Pou5f3, Sox19b and Nanog primes genes for activity during zebrafish genome activation. PLoS Genet. 16:e1008546.

Pastor, W. A., Stroud, H., Nee, K., Liu, W., Pezic, D., Manakov, S., Lee, S. A., Moissiard, G., Zamudio, N., Bourc'his, D., Aravin, A. A., Clark, A. T., and Jacobsen, S. E. 2014. MORC1 represses transposable elements in the mouse male germline. Nat. Commun. 5:5795.

Piper, J., Assi, S. A., Cauchy, P., Ladroue, C., Cockerill, P. N., Bonifer, C., and Ott, S. 2015. Wellington-bootstrap: Differential DNase-seq footprinting identifies cell-type determining transcription factors. BMC Genomics 16:1000.

Quach, B., and Furey, T. S. 2017. DeFCoM: Analysis and modeling of transcription factor binding sites using a motif-centric genomic footprinter. Bioinformatics 33:956-963.

Qutob, D., Tedman-Jones, J., Dong, S., Kuflu, K., Pham, H., Wang, Y., Dou, D., Kale, S. D., Arredondo, F. D., Tyler, B. M., and Gijzen, M. 2009. Copy number variation and transcriptional polymorphisms of Phytophthora sojae RXLR effector genes Avrla and Avr3a. PLoS One 4:e5066.

Rada-Iglesias, A., Bajpai, R., Swigut, T., Brugmann, S. A., Flynn, R. A., and Wysocka, J. 2011. A unique chromatin signature uncovers early developmental enhancers in humans. Nature 470:279-283.
Ramírez, F., Dündar, F., Diehl, S., Grüning, B. A., and Manke, T. 2014. deepTools: A flexible platform for exploring deep-sequencing data. Nucleic Acids Res. 42 (W1):W187-W191.

Roy, S., Kagda, M., and Judelson, H. S. 2013a. Genome-wide prediction and functional validation of promoter motifs regulating gene expression in spore and infection stages of Phytophthora infestans. PLoS Pathog. 9:e1003182.

Roy, S., Poidevin, L., Jiang, T., and Judelson, H. S. 2013b. Novel core promoter elements in the oomycete pathogen Phytophthora infestans and their influence on expression detected by genome-wide analysis. BMC Genomics 14:106.

Seidl, M. F., Wang, R.-P., Van den Ackerveken, G., Govers, F., and Snel, B. 2012. Bioinformatic inference of specific and general transcription factor binding sites in the plant pathogen Phytophthora infestans. PLoS One 7:e51295.

Sheng, Y., Lin, L., Chen, H., Pu, T., Liu, X., Dong, S., Ye, W., and Wang, Y. 2021. The bZIP transcription factor PsBZP32 is involved in cyst germination, oxidative stress response, and pathogenicity of Phytophthora sojae. Phytopathol. Res. 3:1.

Sheng, Y., Wang, Y., Meijer, H. J. G., Yang, X., Hua, C., Ye, W., Tao, K., Liu, X., Govers, F., and Wang, Y. 2015. The heat shock transcription factor PsHSF1 of Phytophthora sojae is required for oxidative stress tolerance and detoxifying the plant oxidative burst. Environ. Microbiol. 17:1351-1364.

Starks, R. R., Biswas, A., Jain, A., and Tuteja, G. 2019. Combined analysis of dissimilar promoter accessibility and gene expression profiles identifies tissue-specific genes and actively repressed networks. Epigenetics Chromatin 12:16.

Sung, M. H., Baek, S., and Hager, G. L. 2016. Genome-wide footprinting: Ready for prime time? Nat. Methods 13:222-228

Sung, M. H., Guertin, M. J., Baek, S., and Hager, G. L. 2014. DNase footprint signatures are dictated by factor dynamics and DNA sequence. Mol. Cell 56:275-285.

Thurman, R. E., Rynes, E., Humbert, R., Vierstra, J., Maurano, M. T., Haugen, E., Sheffield, N. C., Stergachis, A. B., Wang, H., Vernot, B., Garg, K., John, S., Sandstrom, R., Bates, D., Boatman, L., Canfield, T. K., Diegel, M., Dunn, D., Ebersol, A. K., Frum, T., Giste, E., Johnson, A. K., Johnson, E. M., Kutyavin, T., Lajoie, B., Lee, B. K., Lee, K., London, D., Lotakis, D., Neph, S., Neri, F., Nguyen, E. D., Qu, H., Reynolds, A. P., Roach, V., Safi, A., Sanchez, M. E., Sanyal, A., Shafer, A., Simon, J. M., Song, L., Vong, S., Weaver, M., Yan, Y., Zhang, Z., Zhang, Z., Lenhard, B., Tewari, M., Dorschner, M. O., Hansen, R. S., Navas, P. A., Stamatoyannopoulos, G., Iyer, V. R., Lieb, J. D., Sunyaev, S. R., Akey, J. M., Sabo, P. J., Kaul, R., Furey, T. S., Dekker, J., Crawford, G. E., and Stamatoyannopoulos, J. A. 2012. The accessible chromatin landscape of the human genome. Nature 489:75-82.

Tyler, B. M., Tripathy, S., Zhang, X., Dehal, P., Jiang, R. H. Y., Aerts, A., Arredondo, F. D., Baxter, L., Bensasson, D., Beynon, J. L. Chapman, J., Damasceno, C. M. B., Dorrance, A. E., Dou, D., Dickerman, A. W., Dubchak, I. L., Garbelotto, M., Gijzen, M., Gordon, S. G., Govers, F., Grunwald, N. J., Huang, W., Ivors, K. L., Jones, R. W., Kamoun, S. Krampis, K., Lamour, K. H., Lee, M. K., McDonald, W. H., Medina, M. Meijer, H. J. G., Nordberg, E. K., Maclean, D. J., Ospina-Giraldo, M. D. Morris, P. F., Phuntumart, V., Putnam, N. H., Rash, S., Rose, J. K. C. Sakihama, Y., Salamov, A. A., Savidor, A., Scheuring, C. F., Smith, B. M., Sobral, B. W. S., Terry, A., Torto-Alalibo, T. A., Win, J., Xu, Z., Zhang, H., Grigoriev, I. V., Rokhsar, D. S., and Boore, J. L. 2006. Phytophthora genome sequences uncover evolutionary origins and mechanisms of pathogenesis. Science 313:1261-1266.

Wang, Q., Han, C., Ferreira, A. O., Yu, X., Ye, W., Tripathy, S., Kale, S. D., Gu, B., Sheng, Y., Sui, Y., Wang, X., Zhang, Z., Cheng, B., Dong, S., Shan, W., Zheng, X., Dou, D., Tyler, B. M., and Wang, Y. 2011. Transcriptional programming and functional interactions within the Phytophthora sojae RXLR effector repertoire. Plant Cell 23:2064-2086.

Wang, X. W., Lv, J. L., Shi, Y. R., and Guo, L. Y. 2020. Comparative transcriptome analysis revealed genes regulated by histone acetylation and genes related to sex hormone biosynthesis in Phytophthora infestans. Front. Genet. 11:508

Wang, Y., Ye, W., and Wang, Y. 2018. Genome-wide identification of long non-coding RNAs suggests a potential association with effector gene transcription in Phytophthora sojae. Mol. Plant Pathol. 19:2177-2186.

Wood, D. E., and Salzberg, S. L. 2014. Kraken: Ultrafast metagenomic sequence classification using exact alignments. Genome Biol. 15:R46.

Wu, J., Huang, B., Chen, H., Yin, Q., Liu, Y., Xiang, Y., Zhang, B., Liu, B., Wang, Q., Xia, W., Li, W., Li, Y., Ma, J., Peng, X., Zheng, H., Ming, J., Zhang, W., Zhang, J., Tian, G., Xu, F., Chang, Z., Na, J., Yang, X., and Xie, W. 2016. The landscape of accessible chromatin in mammalian preimplantation embryos. Nature 534:652-657. 
Xiang, Q., and Judelson, H. S. 2010. Myb transcription factors in the oomycete Phytophthora with novel diversified DNA-binding domains and developmental stage-specific expression. Gene 453:1-8.

Yan, F., Powell, D. R., Curtis, D. J., and Wong, N. C. 2020. From reads to insight: A hitchhiker's guide to ATAC-seq data analysis. Genome Biol. 21:22.

Yang, C., Ma, L., Xiao, D., Ying, Z., Jiang, X., and Lin, Y. 2019. Integration of ATAC-seq and RNA-Seq identifies key genes in lightinduced primordia formation of Sparassis latifolia. Int. J. Mol. Sci. $21: 185$

Yin, M., Zhang, Z., Xuan, M., Feng, H., Ye, W., Zheng, X., and Wang, Y. 2020. Conserved subgroups of the plant-specific RWP-RK transcription factor family are present in oomycete pathogens. Front. Microbiol. 11:1724.

Yu, G., Wang, L. G., and He, Q. Y. 2015. ChIPseeker: An R/Bioconductor package for ChIP peak annotation, comparison and visualization. Bioinformatics 31:2382-2383.

Zhang, M., Lu, J., Tao, K., Ye, W., Li, A., Liu, X., Kong, L., Dong, S., Zheng, X., and Wang, Y. 2012. A Myb transcription factor of Phytophthora sojae, regulated by MAP kinase PsSAK1, is required for zoospore development. PLoS One 7:e40246.

Zhang, Y., Liu, T., Meyer, C. A., Eeckhoute, J., Johnson, D. S., Bernstein, B. E., Nusbaum, C., Myers, R. M., Brown, M., Li, W., and Liu, X. S 2008. Model-based analysis of ChIP-Seq (MACS). Genome Biol. 9:R137. 